\title{
Conmutación ferroeléctrica y fatiga en cerámicas basadas en $\mathrm{Pb}\left(\mathrm{Zr}_{1-\mathrm{x}} \mathrm{Ti}_{\mathrm{x}}\right) \mathrm{O}_{3}$
}

\author{
J. E. GARCíA, A. RODríGueZ, R. PÉreZ, A. AlbAREDA \\ Departament de Física Aplicada, Universitat Politècnica de Catalunya, \\ c/ Jordi Girona 1-3, mòdul B4, 08034 Barcelona, Spain
}

\begin{abstract}
El proceso de conmutación ferroeléctrica ha sido investigado en cerámicas de composición $\mathrm{Pb}\left(\mathrm{Zr}_{1-\mathrm{x}} \mathrm{Ti}_{\mathrm{x}}\right) \mathrm{O}_{z^{\prime}}$ con $\mathrm{x}=0.40$ y 0.47 , dopadas con $\mathrm{Nb}^{5+}$ o Fe ${ }^{3+}$. Las muestras fueron sometidas a un alto campo eléctrico sinusoidal de baja frecuencia. Los resultados revelaron una fuerte dependencia con el tipo de dopaje. En estado virgen, los materiales con dopaje donador presentan valores mayores de la polarización remanente y de la polarización de saturación que los materiales con dopaje aceptor. Los materiales dopados con $\mathrm{Nb}^{5+}$ muestran un ciclo de histéresis normal, mientras que en los materiales dopados con $\mathrm{Fe}^{3+}$ el ciclo de histéresis muestra un fuerte pinzamiento. Se estudió la variación de las propiedades ferroeléctricas con el número de ciclos aplicados a la muestras. En los materiales con dopaje donador se observa que la fatiga afecta más a los materiales en la frontera de fase morfotrópica que a los materiales en fase romboédrica. Los materiales con dopaje aceptor muestran un típico proceso de desanclaje. En ese tipo de muestras, la reorientación de los defectos complejos produce una rápida eliminación del pinzamiento del ciclo.
\end{abstract}

Palabras clave: conmutación, histéresis, fatiga, PZT.

Ferroelectric switching and fatigue in $\mathrm{Pb}\left(\mathrm{Zr}_{1-\mathrm{x}} \mathrm{Ti}_{x}\right) \mathrm{O}_{3}$-based ceramics.

Polarization switching process was investigated in $\mathrm{Nb}$ - or Fe-doped $\mathrm{Pb}\left(\mathrm{Zr}_{1-\mathrm{x}} \mathrm{Ti}_{\mathrm{x}}\right) \mathrm{O}_{3}$ bulk ceramics, with $\mathrm{x}=0.40$ and 0.47 . High sinusoidal electric field at low frequency was applied to the samples. The results revealed a strong dependence with the kind of doping. At the virgin state, donor-doped materials present higher values of remanent and saturation polarizations than acceptor-doped. Nb-doped materials shown a normal hysteresis loop while Fe-doped materials shown a strong pinching loop. We studied the variation of the remanent polarization and coercive field with the number of hysteresis loops previously performed. In donor-doped samples, it is shown that fatigue affects more to materials at morphotropic phase boundary than at rhombohedral single phase. Acceptor-doped samples showed the expected depinning process. In this case, complex defect reorientation produces a quick elimination of pinching loop.

Keywords: switching, hysteresis, fatigue, PZT.

\section{INTRODUCCIÓN}

Desde hace varias décadas, las cerámicas ferroeléctricas con estructura perovskita están siendo usadas en múltiples aplicaciones. Dentro de este grupo de materiales, las cerámicas basadas en titanato zirconato de plomo (PZT), y especialmente aquellas cuya composición se encuentra en la frontera de fase morfotrópica (MPB), han sido una de las más usadas en aplicaciones tecnológicas como sensores, actuadores y transductores (1-3). Por ello, la naturaleza de los fenómenos que rige el comportamiento de estos materiales ha sido, y es en la actualidad, objeto de innumerables estudios.

Es bien conocido que la adición de dopantes en forma de iones de sustitución modifica notablemente las propiedades macroscópicas del sistema PZT (1). Los dopantes pueden agruparse en donadores o aceptores dependiendo de la valencia del ión que sustituyen cuando son incorporados en la red. Así, la sustitución del $\mathrm{Zr}^{4+} \mathrm{o} \mathrm{Ti}^{4+}$ por un catión trivalente, como el $\mathrm{Fe}^{3+}$, da lugar a la formación de vacancias de oxígeno.
La vacancia de oxígeno y el átomo aceptor introducido en la red forman un defecto complejo con característica dipolar. La interacción de este tipo de defecto con las paredes de dominio dificulta la reorientación de los dominios (4-5). Por otro lado, la sustitución del $\mathrm{Zr}^{4+} \mathrm{o} \mathrm{Ti}^{4+}$ por un catión pentavalente, como el $\mathrm{Nb}^{5+}$, da lugar a la formación de vacancias de plomo y a la vez se inhibe la formación de vacancias de oxígeno, siempre presentes en estos materiales como consecuencias del proceso de síntesis $(4,6)$. La adición de este tipo de dopante disminuye las tensiones mecánicas de la red y también los defectos asociados a las vacancias de oxígeno, favoreciendo la reorientación de los dominios en la dirección del campo aplicado. Como consecuencia, en el sistema PZT se observa diferente respuesta ferroeléctrica en función del tipo de dopaje.

Muchas aplicaciones de los materiales cerámicos ferroeléctricos, como en el caso de los dispositivos de 
memoria, dispositivos electro-ópticos o bien actuadores, están directamente relacionadas con la conmutación ferroeléctrica. En este sentido, la fatiga ferroeléctrica es una de las principales limitaciones de estos materiales en este tipo de aplicaciones. El fenómeno conocido como fatiga puede definirse de manera general como la alteración de las propiedades ferroeléctricas producida por continuas conmutaciones a lo largo del tiempo (7); aunque es común relacionar el término con el decrecimiento de la polarización remanente que experimentan muchos materiales cuando se aplica un alto campo alterno durante un tiempo prolongado. Otros materiales ferroeléctricos experimentan un fenómeno distinto: la polarización remanente aumenta con el número de ciclos. Estos tipos de materiales, como es caso del titanato de bario, presentan un fuerte pinzamiento del ciclo, que ha sido asociado al anclaje de las paredes de dominio producido por defectos de carácter dipolar (8). A este fenómeno, observado en el PZT con dopaje aceptor (9), se le conoce como rejuvenecimiento (deaging) y al efecto que lo provoca se le denomina comúnmente desanclaje (depinning).

El objetivo de este trabajo ha sido, por un lado, estudiar el efecto del tipo de dopaje en las propiedades ferroeléctricas del PZT con composición en la MBP y fuera de ésta y, por otro lado, estudiar la evolución de esas propiedades como una función del número de ciclos continuos realizados a las muestras. Las muestras seleccionadas han permitido evaluar los resultados obtenidos en el marco de los modelos fenomenológicos relacionados con la fatiga, en materiales ferroeléctricos con estructura de tipo perovskita. La causa de las notables diferencias observadas en función del tipo de dopaje, para una misma fase cristalográfica del sistema PZT, puede relacionarse con la presencia de defectos de carácter dipolar producidos por la existencia de vacancias de oxígeno en estos materiales.

\section{EXPERIMENTAL}

Utilizando el método convencional de reacción de estado sólido, se obtuvieron distintas composiciones de titanato zirconato de plomo. A partir de diferentes estequiometrías de $\mathrm{PbO}, \mathrm{Zr}_{2} \mathrm{O}_{3}$ y $\mathrm{TiO}_{2}$ se prepararon composiciones de $\mathrm{Pb}\left(\mathrm{Zr}_{1-\mathrm{x}} \mathrm{Ti}_{\mathrm{x}}\right)$ $\mathrm{O}_{3}$ con $\mathrm{x}=0,40$ (PZT40) y $\mathrm{x}=0,47$ (PZT47). Se adicionó un $1 \%$ en peso de $\mathrm{Nb}_{2} \mathrm{O}_{5}$ o $\mathrm{Fe}_{2} \mathrm{O}_{3^{\prime}}$ de manera que se obtuvieron las composiciones anteriores con dopaje donador y aceptor, respectivamente. La síntesis de las cerámicas se realizó a temperaturas entre $1200-1250^{\circ} \mathrm{C}$, según la composición del material, en una atmósfera saturada de $\mathrm{PbO}$. Se cortaron muestras en forma de disco de $15-16 \mathrm{~mm}$ de diámetro y 0.8-0.9mm de grosor. Después de pulirlas se pintaron electrodos de plata a ambos lados de los discos. Finalmente se les realizó un tratamiento térmico con el objetivo de eliminar solventes orgánicos y relajar las tensiones inducidas en las muestras durante el pulido. El tamaño de grano medio fue estimado entre 3,0-3,5 $\mu \mathrm{m}$ utilizando microscopía electrónica de barrido. El análisis del patrón de difracción de rayos- $X$ reveló la fase cristalográfica esperada: romboédrica para $\mathrm{x}=0,40$ y frontera de fase morfotrópica para $\mathrm{x}=0,47$. No se detectaron fases contaminantes o indeseadas.

La medida de los ciclos de histéresis se realizó utilizando un circuito de tipo Sawyer-Tower, modificado con un medidor de carga. A las muestras se les aplicó una señal sinusoidal de alta tensión y baja frecuencia y se midió directamente la carga. En la figura 1 se muestra un esquema simplificado del sistema experimental. Se utilizado un generador HP33120A y un amplificador Trek663. El sistema se automatizó con el objetivo de medir la histéresis ferroeléctrica en función del número de ciclos.

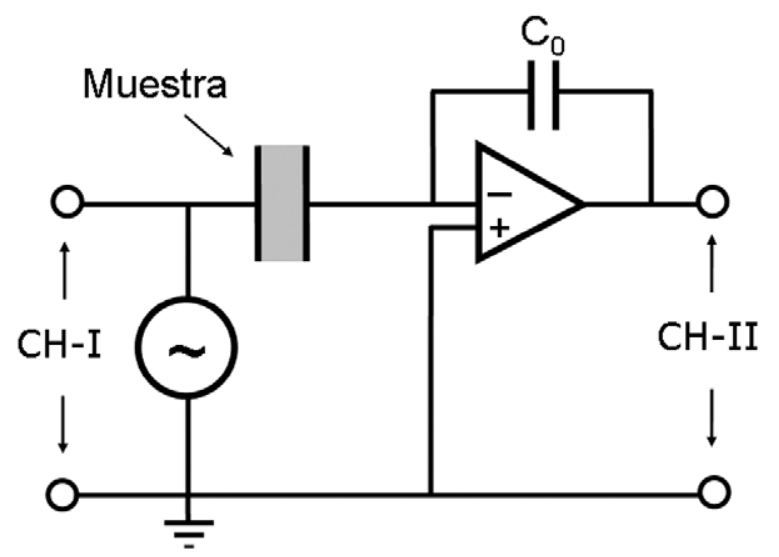

Fig. 1- Esquema simplificado del sistema utilizado para la medida del ciclo de histéresis. Se aplica y se mide una señal sinusoidal de alta tensión a la muestra y se mide el voltaje del condensador de referencia $\mathrm{C}_{0}$.

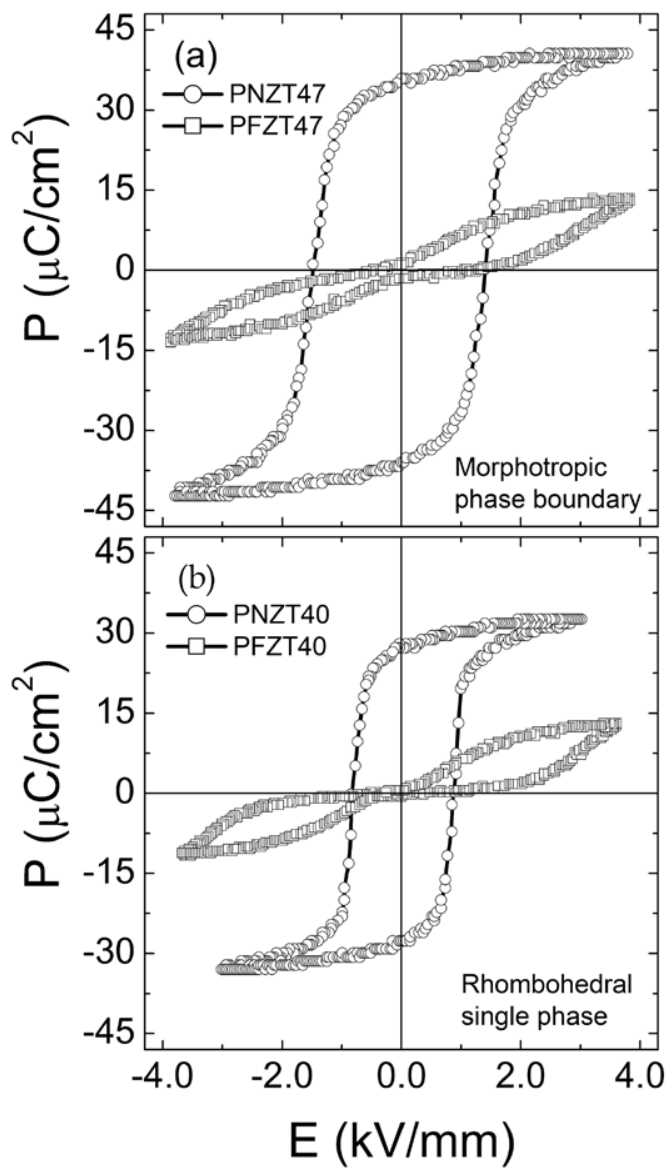

Fig. 2- Ciclos de histéresis en función del dopaje para muestras de una misma fase cristalográfica: (a) frontera de fase morfotrópica; (b) fase romboédrica. Las muestras con dopaje donador (PNZT47 y PNZT40) muestran un ciclo de histéresis normal mientras que las muestras con dopaje aceptor (PFZT47 y PFZT40) muestran un ciclo notablemente pinzado. 

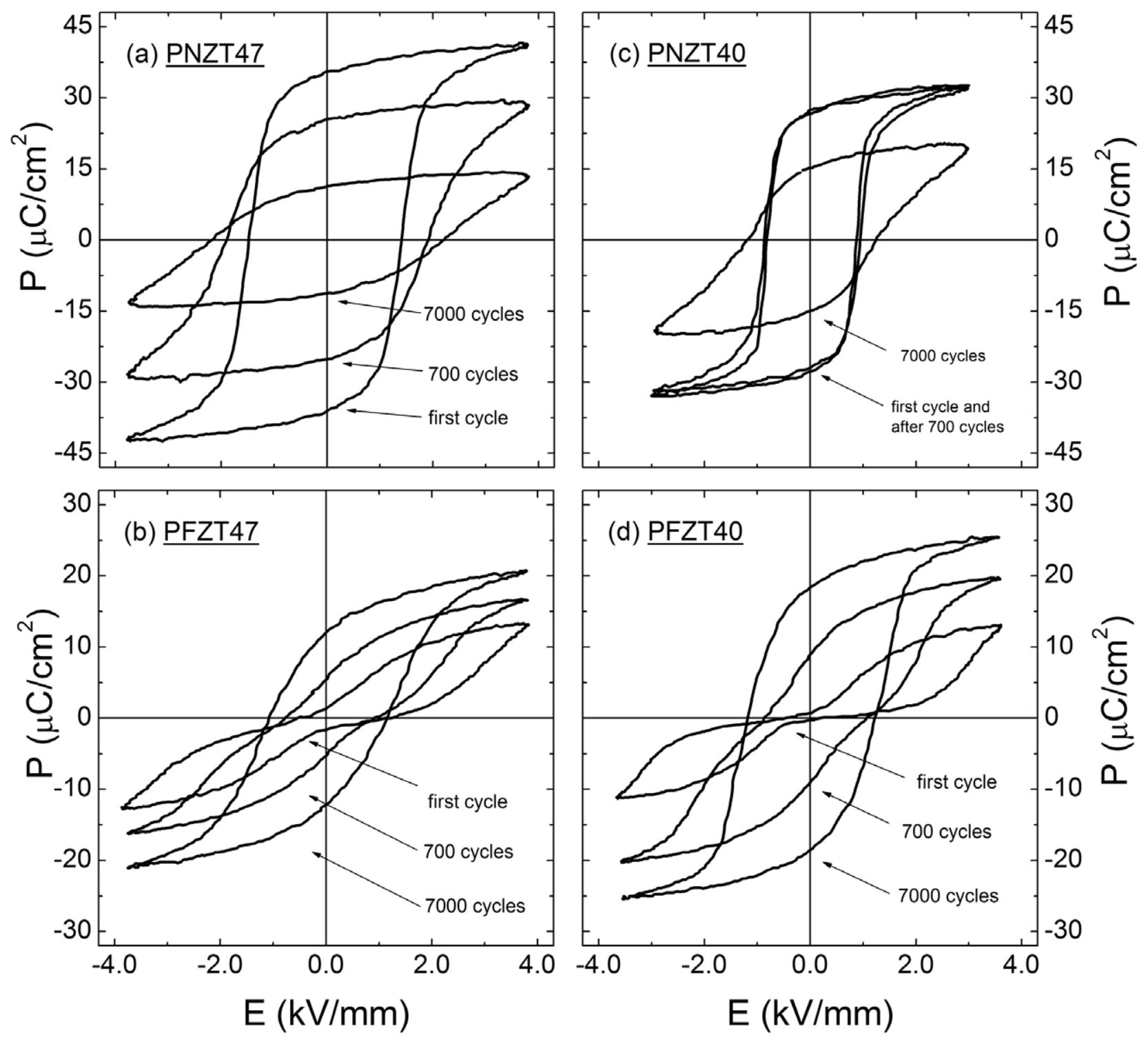

Fig. 3- Histéresis ferroeléctrica después de un determinado número de ciclos en muestras en la frontera de fase (a y b) y en fase romboédrica (c y d). Las muestras dopadas con $\mathrm{Nb}^{5+}$ (a y c) muestran una típico proceso de fatiga mientras que la muestras dopadas con Fe ${ }^{3+}$ (b y d) muestran un proceso de desanclaje.

\section{RESULTADOS}

\subsection{Ciclos de histéresis}

En la figura 2 se muestran los ciclos de histéresis de muestras con composiciones en la frontera de fase morfotrópica [Figura 2(a)], dopadas con $\mathrm{Nb}^{5+}$ (PNZT47) o con $\mathrm{Fe}^{3+}$ (PFZT47), y de muestras romboédricas [Figura 2(b)], también dopadas con $\mathrm{Nb}^{5+}$ (PNZT40) o con $\mathrm{Fe}^{3+}$ (PFZT40). Las medidas se han realizado a $500 \mathrm{mHz}$ a fin de evitar el auto-calentamiento de las muestras (9). Se observa que para un mismo dopaje las propiedades ferroeléctricas no dependen cualitativamente de la fase ferroeléctrica formada. Sin embargo, los resultados revelan que el dopaje tiene una influencia determinante en las propiedades ferroeléctricas de estos materiales.

Las muestras con dopaje donador (PNZT40 y PNZT47) muestran un ciclo típico de histéresis ferroeléctrica, siendo mayor la polarización de saturación, la polarización remanente y el campo coercitivo en la muestra en la MPB que la muestra en fase romboédrica (como era de esperar debido a las excepcionales propiedades del PZT en MPB). Por otro lado, las muestras con dopaje aceptor (PFZT40 y PFZT47) muestran un ciclo de histéresis marcadamente diferente. En ambas composiciones la polarización de saturación es considerablemente menor que en las muestras con dopaje donador y se observa un fuerte pinzamiento del ciclo. Este pinzamiento da lugar a un valor muy bajo de la polarización remanente en relación a la polarización de saturación lo que puede interpretarse como una fuerte pérdida de la polarización de saturación en este tipo de muestras. Los valores, tanto de la polarización de saturación como de la polarización remanente son mayores en la muestra en la MPB que en la muestra en fase romboédrica (ver figura 4 , para $\mathrm{N}=1$ ). 


\subsection{Fatiga}

Con el objetivo de estudiar la evolución de las propiedades ferroeléctricas en el sistema PZT, en función del dopaje y la fase cristalográfica, las muestras fueron sometidas a un alto campo alterno de $500 \mathrm{mHz}$ durante un tiempo prolongado. En la figura 3 se muestra el cambio en la forma del ciclo de histéresis en las muestras estudiadas. Se observa una diferencia notable en la alteración de la polarización remanente con el número de ciclos para uno u otro dopaje; sin embargo no se aprecian diferencias cualitativas en función de la fase cristalográfica para un mismo dopaje. En las muestras dopadas con $\mathrm{Nb}^{5+}$ [Figuras 3(a) y 3(c)] se aprecia una disminución de la polarización remanente en función del número de ciclos. Esta disminución, junto al aumento del campo coercitivo, es más importante en la muestra en la MPB (PNZT47) que en la muestra en fase romboédrica (PNZT40). Puede notarse que una vez aplicados 700 ciclos a ambas muestras, la polarización remanente ha decrecido de forma apreciable en el PNZT47 mientras que en el PNZT40 el ciclo prácticamente no se ha modificado.

Un comportamiento muy diferente al observado en las muestras dopadas con $\mathrm{Nb}^{5+}$ es el que presentan las muestras dopadas con $\mathrm{Fe}^{3+}$. En este tipo de muestras, los resultados revelan un incremento de la polarización remanente con el número de ciclos aplicados a la muestra, produciéndose una rápida eliminación del pinzamiento del ciclo [Figuras 3(b) y 3(d)]. Este efecto contribuye a un notable incremento del campo coercitivo en los primeros ciclos. Puede notarse que el
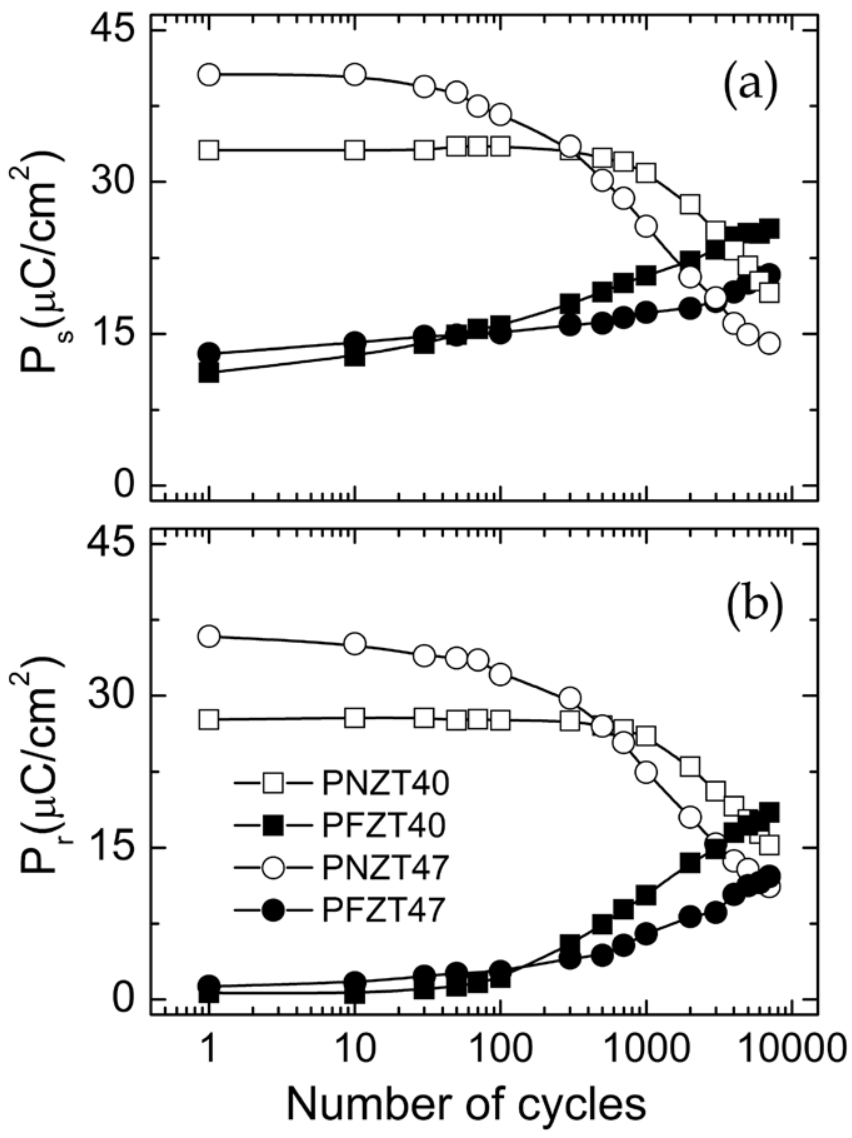

Fig. 4- Evolución de la polarización de saturación (a) y de la polarización remanente (b) en función del número de ciclos en distintas muestras del sistema PZT. incremento de la polarización remanente es más relevante en la muestra romboédrica (PFZT40) que en la muestra en la MPB (PFZT47), a pesar de que el pinzamiento del ciclo del PFZT40 es mayor que el del PFZT47 cuando ambas se encuentran en estado virgen (primer ciclo).

Para complementar los resultados anteriores, en la figura 4 se muestra la evolución de la polarización de saturación (a) y de la polarización remanente (b) con el número de ciclos en las muestras estudiadas. Puede observarse como para las muestras con dopaje donador tanto la polarización remanente como la de saturación decrecen con el número de ciclos. Ese decrecimiento es notablemente mayor en la muestra en la MBP que en la muestra romboédrica. En el PNZT47, tanto la polarización remanente como la de saturación decrecen un 10\% después de unos 100 ciclos, mientras que en el PNZT40 se necesitan algo más de 1000 ciclos para el mismo porcentaje de decrecimiento.

A diferencia de lo observado en las muestras con dopaje donador, en las muestras con dopaje aceptor tanto la polarización remanente como la de saturación se incrementan con el número de ciclos. Este efecto es más importante en la polarización remanente que en la polarización de saturación debido al pinzamiento del ciclo cuando las muestras se encuentran en estado virgen. En el PFZT47, por ejemplo, después de 1000 ciclos la polarización remanente es 5 veces la del primer ciclo mientras que la de saturación solo se incrementa un $30 \%$. Tal como se apreciaba en la figura 3, tanto el incremento de la polarización remanente como de la polarización de saturación es mayor en el PFZT40 que en el PFZT47, de manera que después de 7000 ciclos las propiedades ferroeléctricas de la muestra romboédrica con dopaje aceptor no difiere de forma cualitativa con el ciclo de histéresis de una muestra con dopaje donador en estado virgen.

\section{DISCUSIÓN}

El efecto del proceso de envejecimiento en las propiedades ferroeléctricas en cerámicas de tipo perovskita fue descrito por Jonker en 1972 (8). Una muestra "joven" (justo después de ser enfriada desde una temperatura superior al punto de Curie) de $\mathrm{BaTiO}_{3}$ o $\mathrm{Ba}\left(\mathrm{Ti}_{0.95} \mathrm{Zr}_{0.05}\right)_{3}$ exhibe un ciclo de histéresis "normal"; sin embargo, con el paso del tiempo el material evoluciona hacia un estado estable donde se observa un notable pinzamiento del ciclo. Este hecho ha sido atribuido a la gradual orientación de los defectos complejos de carácter dipolar formados por impurezas y vacancias. Esos defectos, orientados en la dirección del eje polar, actúan como centros de anclaje de las paredes de dominio dando lugar a una disminución de la polarización remanente (10). Estudios recientes en titanato zirconato de plomo dopado con potasio en sustitución del plomo (PKZT), demuestran que el pinzamiento del ciclo está relacionado con los defectos complejos asociados a la formación de vacancias de oxígeno por la adición del potasio como dopaje aceptor (11).

Los resultados obtenidos en este trabajo muestran un marcado pinzamiento en ciclo de histéresis del PFZT que resulta ser cualitativamente independiente de la fase cristalográfica. En estos materiales, el dopaje introducido genera vacancias de oxígeno que forman defectos complejos de carácter dipolar, de la misma forma que en el caso del PKZT. Por otro lado, los resultados revelan un ciclo de histéresis normal en el caso del PNZT. En estos materiales, la adición de dopante 


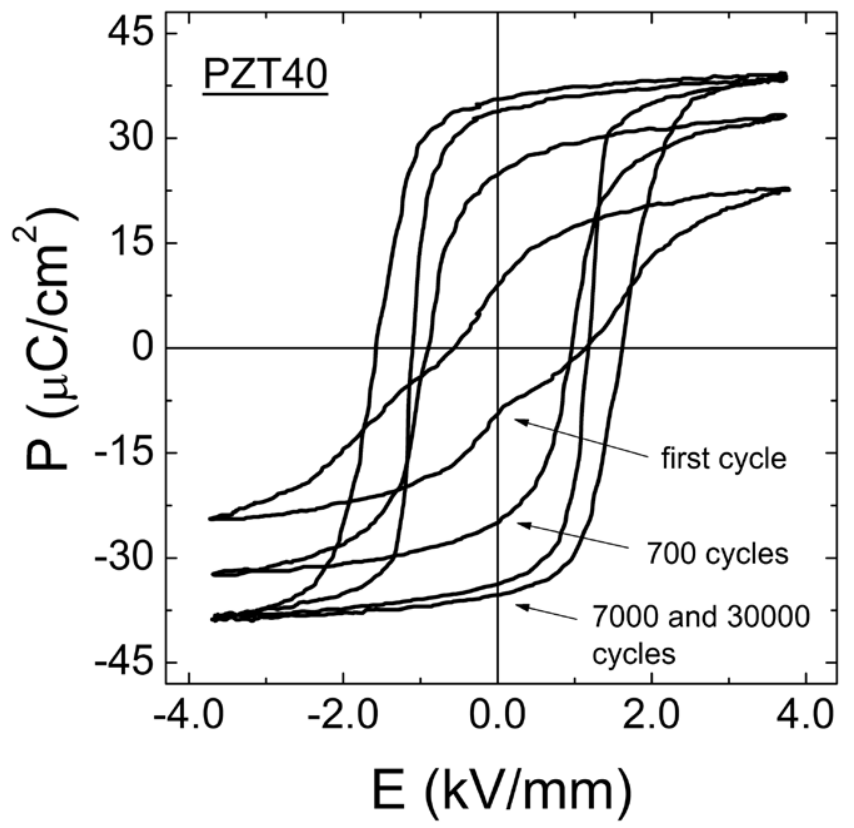

Fig. 5- Histéresis ferroeléctrica después de un determinado número de ciclos en una muestra de PZT puro en fase romboédrica.

donador inhibe la formación de vacancias de oxígeno por compensación de carga, por tanto no es de esperar presencia de defectos asociados a dichas vacancias (6). Estos resultados permiten afirmar que en efecto, la presencia de defectos por complejos asociados a la formación de vacancias de oxígeno es la causa del pinzamiento del ciclo de histéresis observado en el PZT con dopaje aceptor.

Con el objetivo de complementar los resultados, se midió el ciclo de histéresis en un PZT puro en fase romboédrica (PZT40). En la figura 5 se observa que el ciclo de histéresis de este material en estado virgen también muestra pinzamiento. Es bien conocido que en el PZT puro la formación de vacancias de oxígeno debido a la evaporación de plomo en el proceso de síntesis es inevitable. Por tanto, en los materiales libres de dopantes también se forman defectos complejos asociados a dichas vacancias (12-13). Es de esperar que la cantidad de defectos presentes en el PZT40 sea inferior al PFZT, lo cual explicaría las diferencias cuantitativas en las características del ciclo entre ambos materiales. Los resultados obtenidos en el PZT puro corroboran que el anclaje de las paredes de dominio, producido por los defectos de carácter dipolar, asociados a las vacancias de oxígeno, son en realidad los responsables del pinzamiento del ciclo de histéresis en el sistema PZT. Es posible afirmar que el dopaje aceptor es responsable, únicamente, de un incremento en el pinzamiento.

El efecto que produce en las propiedades ferroeléctricas del PZT la aplicación de un alto campo eléctrico sinusoidal durante un largo periodo de tiempo ha sido estudiado, entre otros autores, por Lente et. al., en cerámicas con composición en la MPB y distinto tipo de dopaje $(9,10)$. En el PNZT se observa una disminución gradual de la polarización remanente y la polarización de saturación, mientras que en el PFZT se observa un aumento de esas magnitudes. La fatiga observada en el PNZT se supone debida a la migración de las cargas de espacio hacia las fronteras de dominio, lo cual produce un gradual anclaje de las paredes de dominio dificultándose la conmutación ferroeléctrica $(9,14)$. Por otro lado, el efecto de desanclaje (depinning) observado en el PFZT, se supone debido a la reorientación de los defectos complejos presentes en este tipo de materiales. La aplicación de una campo alterno desencadena un proceso de desorientación de los defectos complejos provocando una disminución de la interacción paredes de dominio - defectos complejos. Este hecho es la causa de la disminución del anclaje de las paredes de dominio dando lugar un aumento gradual de las polarizaciones remanente y de saturación $(4,9)$.

En este trabajo se obtienen resultados similares a los reportados por Lente et. al. (10), pero en este caso también en materiales con composición fuera de la MPB. A pesar de que los resultados obtenidos resultan ser cualitativamente independientes de la fase cristalográfica, existen diferencias cuantitativas en la evolución de la fatiga en una u otra fase. Este proceso afecta menos a las muestras en fase romboédrica que a las muestras en la frontera de fase morfotrópica. Un resultado similar fue obtenido en muestras de PZT dopadas con lantano (PLZT), donde para un determinado número de ciclos, las muestras romboédricas no se ven afectadas por la fatiga mientras que el valor de la polarización remanente en las muestras con composición en la MPB experimentan un fuerte decrecimiento (15). Este hecho es atribuido, por un lado, a que las muestras romboédricas presentan un menor campo coercitivo y un menor estrés interno (del orden del $0.5 \%$ para la celda romboédrica en comparación con el $2 \%$ en la tetragonal), lo cual facilita el proceso de conmutación y retrasa el proceso de fatiga (15).

Con el objetivo de corroborar la influencia de los defectos asociados a las vacancias de oxígeno, se muestra en la figura 5 la evolución de las propiedades ferroeléctricas del PZT puro con el número de ciclos. Se observa como, en este material, ocurre un proceso de desanclaje, indicando que la presencia de defectos complejos asociados a las vacancias de oxígeno son determinantes en la respuesta del PZT a un campo alterno prologado en el tiempo. En esta muestra el campo se aplicó durante un tiempo mayor (hasta algo más de 30 mil ciclos) y se observó que después de los 7000 ciclos la polarización remanente tiende a saturarse mientras el campo coercitivo continúa aumentando. Estudios posteriores servirán para aclarar la evolución de las propiedades para un número mayor de ciclos.

\section{CONCLUSIONES}

En este trabajo se ha estudiado el proceso de conmutación ferroeléctrica en el sistema PZT. Se investigaron muestras en fase romboédrica y en la frontera de fase morfotrópica, en ambos casos con dopaje aceptor $\left(\mathrm{Fe}^{3+}\right)$ o dopaje donador $\left(\mathrm{Nb}^{5+}\right)$. Los ciclos de histéresis evidenciaron una fuerte dependencia con el tipo de dopaje que resultó ser cualitativamente independiente de la fase cristalográfica. Los resultados muestran que el fuerte pinzamiento del ciclo presente en las muestras con dopaje aceptor puede ser debido al efecto de anclaje de las paredes de dominio producido por la interacción de dichas paredes con los defectos complejos asociados a las vacancias de oxígeno. El ciclo de histéresis en un PZT libre de dopaje corrobora dicha afirmación.

La evolución de las propiedades ferroeléctricas cuando las muestras fueron sometidas a un campo alterno continuo también evidenció una fuerte dependencia con el tipo de dopaje. En las muestras con dopaje donador se observa una disminución gradual de las polarizaciones remanente y de saturación y un aumento del campo coercitivo. Este efecto, 
conocido como fatiga, se supone debido a la migración de cargas de espacio a la frontera de los dominios ferroeléctricos, lo cual dificulta el movimiento de las paredes de dominio y por tanto la reorientación de los dominios con el campo. Se observó que la fatiga afecta más a las muestras en la frontera de fase morfotrópica que a las muestras romboédricas. Este hecho es debido a que el menor campo coercitivo y el menor estrés interno de la muestra romboédrica facilita el proceso de reorientación de la polarización, retrasando el proceso de fatiga en relación a las muestra en la frontera de fase morfotrópica.

En las muestras con dopaje aceptor se observa el fenómeno conocido como desanclaje. Las polarizaciones remanente y de saturación aumentan con el número de ciclos debido a la desorientación de los defectos complejos al aplicar un campo alterno continuo. Ese efecto provoca una disminución en la interacción de los defectos complejos con las paredes de dominio facilitando el proceso de conmutación ferroeléctrica. La influencia de los defectos asociados a las vacancias de oxígeno fue demostrada a partir de los resultados obtenidos en el PZT libre de dopaje, donde también se produce un proceso de desanclaje.

\section{AGRADECIMIENTOS}

Este trabajo ha sido realizado con el apoyo del proyecto español MAT2004-01341 (MEC). Un especial agradecimiento al Prof. José A. Eiras, de la Universidade Federal de Sao Carlos, Brasil, por suministrar las muestras.

\section{BIBLIOGRAFÍA}

1. B. Jaffe, W. R. Cook, and H. Jaffe, Piezoelectric ceramics, Academic Press, New York (1971).

2. L. E. Cross, Ferroelecric materials for electromechanical transducer applications, Jpn. J. Appl. Phys., 34, 2525-2532 (1995).

3. G. H. Haertling, Ferroelectric ceramics: history and technology, J. Am. Ceram. Soc., 82, 797-818 (1999).

4. S. Takahashi, Effects of impurity doping in lead zirconate-titanate ceramics, Ferroelectric, 41, 277-290 (1982).

5. W. L. Warren, G. E. Pike, K. Vanheusden, D. Dimos, B. A. Tuttle, and J. Robertson, Defect-dipole alignment and tetragonal strain in ferroelectrics, J. Appl. Phys., 79, 9250-9257 (1996).

6. Z. Zhang, L. Lu, C. Shu, and P. Wu, Computational investigation of B-site donor doping effect on fatigue behavior of lead zirconate titanate, Appl. Phys. Lett., 89, 152909 (2006).

7. D. C. Lupascu, Fatigue in ferroelectric ceramics and related issues, SpringerVerlag, Germany (2004).

8. G. H. Jonker, Nature of aging in ferroelectric ceramics, J. Am. Ceram. Soc., 55, 57-58 (1972).

9. M. H. Lente and J. A. Eiras, Interrelationship between self-heating and ferroelectric properties in PZT ceramics during polarization reorientation, J. Phys.: Condens. Matter, 12, 5939-5949 (2000).

10. M. H. Lente and J. A. Eiras, Domain reorientation anisotropy in ferroelectric polycrystals, J. Appl. Phys., 92, 2112-2117 (2002).

11. Q. Tan, J. Li, and D. Viehland, Role of lower valent substituent-oxygen vacancy complexes in polarization pinning in potassium-modified lead zirconate titanate, Appl. Phys. Letter, 75,418-420 (1999).

12. G. Burns and B. A. Scott, Raman spectra of polycrystalline solids: application to $\mathrm{PbTi}_{1-\mathrm{x}} \mathrm{Zr}_{\mathrm{x}} \mathrm{O}_{3}$ system, Phys. Rev. Lett., 25, 1191-1194 (1970).

13. C. H. Park and D. J. Chadi, Microscopic study of oxygen-vacancy defects in ferroelectric perovskites, Phys. Rev. B, 57, R13961 (1998).

14. Q. Y. Jiang and L. E. Cross, Effects of porosity on electric fatigue behavior in PLZT and PZT ferroelectric ceramics, J. Mat. Sci., 28, 4536-4543 (1993).

15. Q. Y. Jiang, E. C. Subbarao, and L. E. Cross, Effect of composition and temperature on electric fatigue of La-doped lead zirconate titanate ceramics, J. Appl. Phys., 75, 7433-7443 (1994). 